\title{
Reduced Anterior Temporal and Hippocampal Functional Connectivity During Face Processing Discriminates Individuals with Social Anxiety Disorder from Healthy Controls and Panic Disorder, and Increases Following Treatment
}

\author{
Spiro P Pantazatos*, 1,2,9, Ardesheer Talati ${ }^{3,4,9}$, Franklin R Schneier ${ }^{3,5}$ and Joy Hirsch ${ }^{*, 1,6,7,8}$ \\ ' fMRI Research Center, Columbia University Medical Center, New York, NY, USA; ${ }^{2}$ Department of Physiology and Cellular Biophysics, Columbia \\ University, New York, NY, USA; ${ }^{3}$ Department of Psychiatry, Columbia University, New York, NY, USA; ${ }^{4}$ Division of Epidemiology, New York State \\ Psychiatric Institute, New York, NY, USA; ${ }^{5}$ Anxiety Disorders Clinic, New York State Psychiatric Institute, New York, NY, USA; ${ }^{6}$ Department of \\ Neuroscience, Columbia University, New York, NY, USA; ${ }^{7}$ Departments of Radiology, Columbia University, New York, NY, USA; ${ }^{8}$ Department of \\ Psychology, Columbia University, New York, NY, USA
}

\begin{abstract}
Group functional magnetic resonance imaging ( $\mathrm{MMRI}$ ) studies suggest that anxiety disorders are associated with anomalous brain activation and functional connectivity (FC). However, brain-based features sensitive enough to discriminate individual subjects with a specific anxiety disorder and that track symptom severity longitudinally, desirable qualities for putative disorder-specific biomarkers, remain to be identified. Blood oxygen level-dependent (BOLD) FMRI during emotional face perceptual tasks and a new, large-scale and condition-dependent FC and machine learning approach were used to identify features (pair-wise correlations) that discriminated patients with social anxiety disorder $(S A D, N=16)$ from controls $(N=19)$. We assessed whether these features discriminated $S A D$ from panic disorder (PD, $N=16$ ), and SAD from controls in an independent replication sample that performed a similar task at baseline $(\mathrm{N}: \mathrm{SAD}=15$, controls $=17)$ and following 8 -weeks paroxetine treatment $(\mathrm{N}: \mathrm{SAD}=12$, untreated controls $=7)$. High $\mathrm{SAD}$ vs HCs discrimination (area under the ROC curve, AUC, arithmetic mean of sensitivity and specificity) was achieved with two FC features during unattended neutral face perception $(A \cup C=0.88, P<0.05$ corrected). These features also discriminated $S A D$ VS PD (AUC $=0.82$, $P=0.000 \mathrm{I}$ ) and $\mathrm{SAD}$ vs $\mathrm{HCs}$ in the independent replication sample (FC during unattended angry face perception, $A \cup C=0.7 \mathrm{I}$, $P=0.01$ ). The most informative FC was left hippocampus-left temporal pole, which was reduced in both SAD samples (replication sample $P=0.027$ ), and this $F C$ increased following the treatment (post $>$ pre, $t_{(1 I)}=2.9, P=0.007$ ). In conclusion, $S A D$ is associated with reduced FC between left temporal pole and left hippocampus during face perception, and results suggest promise for emerging FC-based biomarkers for SAD diagnosis and treatment effects.
\end{abstract}

Neuropsychopharmacology (2014) 39, 425-434; doi: 10.1038/npp.2013.21 I; published online 2 October 2013

Keywords: diagnostic classification; selective serotonin reuptake inhibitor; SSRI; support vector machine; longitudinal; neuroimaging

\section{INTRODUCTION}

There is an increasingly recognized need for biomarkers in neuro-degenerative and psychiatric disorders for both early and differential diagnosis, personalized prediction of treatment response, and treatment and drug discovery (Gordon and Koslow, 2010). Biomarker research for anxiety

*Correspondence: Dr SP Pantazatos, Department of Physiology and Cellular Biophysics, Columbia University, 1051 Riverside Dr, Room 570 I, New York, NY 10032, USA, Tel: + 734678 6792, Fax: + I 212 543-65 I5, E-mail: spiropan@gmail.com or Professor Joy Hirsch, Yale School of Medicine, 300 George St, Suite 902, New Haven, CT 065 I I, USA, Fax: + I 203-737-8095, E-mail: joy.hirsch@yale.edu

${ }^{9}$ These authors contributed equally to this work and should be viewed as co-first authors.

Received 31 May 2013; revised 14 August 2013; accepted 14 August 2013; accepted article preview online 20 August 2013 disorders has received relatively little attention, despite the fact they are the most common psychiatric condition, with a lifetime prevalence of $29 \%$ (Kessler et al, 2005). Social anxiety disorder (SAD) is among the most common of these disorders (Jefferys, 1997), with a 7-13\% lifetime prevalence (Kessler et al, 1994). SAD is characterized by heightened anxiety and avoidance during social interactions. It has an early onset ( $80 \%$ of cases occur before age 18 years) (Otto et al, 2001), and often precedes other anxiety, mood, and substance abuse/dependence disorders (Lampe et al, 2003; Randall et al, 2001). SAD is associated with a significant functional impairment and distress in work and social domains and usually persists unless treated (Schneier et al, 1994; Lochner et al, 2003).

Models of SAD (Clark and McManus, 2002; Rapee and Heimberg, 1997) have highlighted the role of sensitivity to perceived social threats, which is triggered by biased 
appraisals of social situations. These maladaptive appraisals transform innocuous social cues into interpersonal threats that induce a cascade of fears of negative evaluation, somatic concerns, inhibited behavior, and negative emotional reactivity. Behavioral studies have shown a negative interpretation bias in $\mathrm{SAD}$, such that ambiguous facial expressions are more likely to be deemed as threatening (Veit et al, 2002; Yoon and Zinbarg, 2008a; Mohlman et al, 2007). Neuroimaging studies suggest exaggerated limbic responses to harsh or angry faces (Stein et al, 2002) as well as neutral faces (Gentili et al, 2008) in SAD, while a recent review of implicit face processing tasks in SAD suggests amplified responses to faces in general (Schulz et al, 2013) despite heterogeneity of findings for emotion specificity and activated brain regions. A meta-analysis of neuroimaging studies of anxiety (using tasks that mostly involved emotional face viewing) found overall greater activation of amygdala and insula, structures linked to negative emotional response, in SAD subjects relative to matched comparison subjects (Etkin and Wager, 2007).

Neuroimaging studies have typically used emotional face paradigms to contrast average activation between patients and healthy control (HC) subjects, identifying significant differences in particular brain regions. However, clinical application of neuroimaging for the diagnosis and treatment of anxiety would require a quantitative measure of brain activity that can distinguish single patients with a specific disorder (eg, SAD) from healthy individuals as well as from individuals with a related disorder (eg, panic disorder, PD, which is characterized by panic attacks and anxiety symptoms that overlap those of SAD but are not exclusively related to social stimuli). To accomplish this, we shifted the focus of our data analysis from average differences (or similarities) in regional brain activity between groups to features of brain activity that maximize the probability of predicting the correct diagnosis within a single subject.

A novel approach based on multivariate machine learning-based pattern analysis of large-scale, condition-dependent functional connectivity (FC) recently demonstrated increased sensitivity of patterns of interactivity (ie, pairwise FC from hundreds of nodes) relative to patterns of activity (ie, beta or contrast activation maps) in predicting subliminal and unattended viewing of fearful $v s$ neutral faces in healthy subjects: functional connections that discriminated supraliminal fearful from neutral faces included amygdala, fusiform, thalamus, superior temporal sulcus, superior occipital cortex, hippocampus, angular gyrus, and cerebellum (Pantazatos et al, 2012b) whereas FC that discriminated subliminal fear from neutral faces included middle temporal gyrus, angular gyrus, cerebellum, superior frontal gyrus, and amygdala (Pantazatos et al, 2012a). On the basis of this and previous evidence that SAD subjects exhibit anomalies in the cognitive-emotional processing of emotional and ambiguous social stimuli, it was hypothesized that patterns of interactivity during fearful and neutral face processing would be sensitive in discriminating whether an individual subject has SAD. The current work examined (1) whether pattern classification of FC during unattended or subliminal processing of emotional or neutral faces can predict SAD diagnosis (control $v s$ $\mathrm{SAD}$, and SAD vs PD), (2) whether discriminating features from the above were also replicated in an independent sample, and (3) whether these same discriminating features tracked symptom severity longitudinally in both HCs and subjects that underwent 8 weeks of treatment with the selective serotonin reuptake inhibitor (SSRI) paroxetine. A treatment/longitudinal component of our study was included to test whether features that predict cross-sectional diagnosis are sensitive to changes in symptom severity, and hence may be potential neurobiological markers of disease state progression and/or response to treatment interventions. A secondary objective compared the classification performance achieved when using interactivity (pair-wise correlations) vs activity (ie, beta estimates from SPM maps). If FC-based features can be shown to reliably categorize subjects with a diagnosis of SAD, discriminate them from subjects with a closely related disorder such as PD, and demonstrate a tendency to normalize following treatment, then this would represent an important advance in the development of biomarkers for psychiatric diagnosis and treatment effects.

\section{MATERIALS AND METHODS}

\section{Ethics Statement}

All procedures and tasks were reviewed for ethical concerns and protection of human subjects by the Columbia University and New York State Psychiatric Institute Institutional Review Boards before subject recruitment and data collection.

\section{Subjects}

Primary sample. Twenty HC subjects, eighteen subjects (ages 18-50) diagnosed with SAD, and sixteen patients diagnosed with $P D$ were recruited through web advertisements (except for seven of the SAD subjects recruited from a genetic study of anxiety; Talati et al, 2008). Functional scans of two subjects (one control and one SAD) were unusable due to scanner technical issues, while a third subject was excluded because she was diagnosed with both SAD and PD. Recruitment and clinical procedures have been detailed elsewhere (Talati et al, 2013) and are described in more detail in Supplementary Methods.

Replication sample. Eighteen medication-free adults with a primary diagnosis of GSAD (age 20-52) and seventeen age, sex, and race-matched HCs were recruited through media notices and clinical referrals. Diagnoses were based on the psychiatric interview and confirmed by the Structured Clinical Interview for DSM-IV Axis I disorders. Data from four GSAD patients were excluded from analyses due to technical issues, yielding $14 \mathrm{GSAD}$ patients and $17 \mathrm{HCs}$ used for diagnostic classification analysis. Twelve GSAD patients and seven HCs completed a second scan following the 8-week paroxetine treatment (or non-treatment for HCs). See Schneier et al (2011) and Supplementary Methods for more details regarding subjects, recruitment, and treatment. Due to the small number of HCs that completed a second scan they were not included in longitudinal analyses (however, see Supplementary Results for provisionary analyses that include these subjects). 


\section{Behavioral Task}

Primary sample. Subjects performed a previously described task from our group (Etkin et al, 2004; Pantazatos et al, 2012b), which consists of color identification of Ekman fearful, neutral, masked fearful, and masked neutral faces (F, N, MF, and $\mathrm{MN}$, respectively) within a blocked paradigm (four $20 \mathrm{~s}$ blocks for each condition, $15 \mathrm{~s}$ baseline between each block). See Supplementary Methods for further details regarding the task paradigm and stimuli.

Replication sample. Subjects performed gender identification of angry, happy, and neutral faces $(\mathrm{A}, \mathrm{H}$, and $\mathrm{N}$, respectively) drawn from the same standard series as above (Ekman and Friesen, 1976), and within a blocked paradigm (four $20 \mathrm{~s}$ blocks for each condition, 12-14 s baseline between each block). See Supplementary Methods for further details

\section{Image Acquisition and Analyses}

fMRI acquisition. Functional data were acquired on a 1.5 Tesla GE Signa MRI scanner in the functional MRI Research Center at Columbia University Medical Center, using a gradient-echo, $\mathrm{T} 2{ }^{*}$-weighted echoplanar imaging (EPI) with blood oxygen level-dependent (BOLD) contrast pulse sequence. Twenty-four contiguous axial slices were acquired along the AC-PC plane, with a $64 \times 64$ matrix and $20 \mathrm{~cm}$ field of view (voxel size $3.125 \times 3.125 \times 4 \mathrm{~mm}, \mathrm{TR}=2000, \mathrm{TE}=40$, flip angle $=60$ ). Structural data were acquired using a 3D T1weighted spoiled gradient recalled (SPGR) pulse sequence with isomorphic voxels $(1 \times 1 \times \mathrm{mm})$ in a $24 \mathrm{~cm}$ field of view $(256 \times 256$ matrix, $\sim 186$ slices, $\mathrm{TR}=34 \mathrm{~ms}, \mathrm{TE}=3 \mathrm{~ms})$.

GLM analysis. Functional data were preprocessed and processed in SPM8 (Wellcome Department of Imaging Neuroscience, London, UK). For preprocessing, the realigned $\mathrm{T} 2{ }^{*}$-weighted volumes were slice-time corrected, spatially transformed, and resampled to a standardized brain (Montreal Neurologic Institute, $2 \times 2 \times 2 \mathrm{~mm}^{3}$ cube resolution) and smoothed with a 8-mm full-width half-maximum Gaussian kernel. First-level regressors were created by convolving the onset of each condition (primary sample: MF, MN, F, and N, replication sample: $\mathrm{A}, \mathrm{H}$, and $\mathrm{N}$ ) with the canonical HRF with duration of $20 \mathrm{~s}$. Additional nuisance regressors included six motion parameters, white matter and csf signal, which were removed before time-series extraction. For the current work, the same GLM analysis served two main purposes: (1) facilitate removal of nuisance effects from time series before FC estimation using structurally (atlas-based) defined ROIs, and (2) produce beta estimates of each condition for case $v s$ control classification analyses (primary sample) using spatial activity patterns.

FC estimation. Atlas-based parcellation (see Supplementary Figure 1) was applied and pair-wise correlations between 248 nodes (derived from 124 atlas-based brain regions) were computed using 40 total time points of fMRI data that were segmented and concatenated from four conditions; unattended and non-masked (ie, unattended) F and $\mathrm{N}$ faces, and subliminal, $\mathrm{MF}$ and $\mathrm{MN}$ faces (see Pantazatos et al, 2012b and Pantazatos et al, 2012a and
Supplementary Methods for more details and analysis schematic). Correlations over the full run were also computed (Full). This resulted in 30628 total functional connections (z-transformed Pearson's correlations) for each condition of interest (F, N, MF, MN, and Full), which were used as features for diagnostic classification.

Pattern analysis of large-scale FC to predict SAD diagnosis. For all binary classification tasks, a linear kernel SVM (Vapnik, 1999) with a filter feature selection $(t$-test) and leave-one-out cross-validation was used. During each iteration of leave-one-out cross-validation (primary sample), one subject was withheld from the data set and (1) a 2-sample $t$-test was performed over the remaining training data, (2) the features were ranked by absolute $t$-score and the top $\mathrm{N}$ were selected, (3) these selected features were then used to predict the class of the withheld test examples during the classification stage. For classification in the replication sample, the SVM model was learned from the whole primary sample using the top two features identified in the analysis above, and this same model was used to predict SAD vs controls in the replication sample. Before learning, the effects of age and gender were regressed out from the features using a general linear model, and features were z-scored. Classification, performance assessment, and confidence interval estimation followed previously described procedures (Pantazatos et al, 2012a, 2012b), and are further described in Supplementary Methods.

Univariate replication analyses. Features identified in the primary analysis were subjected to univariate statistical tests in the replication sample. SAD vs HC (pre-treatment) was assessed with the Mann-Whitney $U$-test, pre-post group changes in FC were assessed using the paired $t$-test, and longitudinal pre-post correlations (decrease $\triangle$ LSAS vs increase $\Delta \mathrm{FC}$ ) were assessed using Spearman's Rho (rank correlation coefficient). Our hypotheses were based on the directions observed in the primary sample, so reported $P$-values are one-tailed.

\section{RESULTS}

\section{Behavioral Results}

The average response rates in the color discrimination task were: $\operatorname{HCs} 96.4 \%(\sigma=6.2 \%)$, SAD $99.9 \%(\sigma=0.15 \%)$, and PD $98.1 \%(\sigma=4.2 \%)$. Mean accuracies were HCs $96.3 \%$ $(\sigma=4.4 \%), \quad \mathrm{SAD} \quad 99.1 \% \quad(\sigma=0.96 \%)$, and $\mathrm{PD} \quad 97.0 \%$ $(\sigma=4.3 \%)$. Mean reaction times were: HCs $0.66 \mathrm{~s}(\sigma=0.09 \mathrm{~s})$, SAD $0.64 \mathrm{~s}(\sigma=0.16 \mathrm{~s})$, and PD $0.70 \mathrm{~s}(\sigma=0.11 \mathrm{~s})$, indicating that subjects performed the color discrimination task as instructed. The groups did not differ in $\mathrm{RT}\left(\mathrm{F}_{50}=1.75\right.$, $P=0.18)$ or accuracy $\left(\mathrm{F}_{47}=0.46, P=0.63\right.$, one-way ANOVA $)$.

\section{Discriminating Between SAD and HCs with Patterns of FC}

For SAD $(n=16)$ vs HC $(n=19)$ classification in the primary sample (see Table 1 for demographics), a peak area under the ROC curve (AUC) of $0.88(P<0.004$ corrected, $0.81,1.090 \% \mathrm{CI}$ ) was achieved when learning was based on the top two features in each training set, derived from the $\mathrm{N}$ 
Table I Demographics Table

\begin{tabular}{|c|c|c|c|c|}
\hline & SAD & PD & Control & Statistic \\
\hline \multicolumn{5}{|l|}{ Cross-sectional } \\
\hline Primary sample & $N=16$ & $N=16$ & $N=19$ & \\
\hline Age (mean years, SD) & $33.6(7.1)$ & $32.2(11)$ & $31.7(8)$ & $F(2,49)=0.21, P=0.81$ \\
\hline Gender (number, \% female) & $14(88 \%)$ & $12(75 \%)$ & $8(42 \%)$ & ChiSq $=8.7, P=0.01$ \\
\hline LSAS score (mean, SD) & $\mathrm{n} / \mathrm{a}$ & $\mathrm{n} / \mathrm{a}$ & $\mathrm{n} / \mathrm{a}$ & \\
\hline Replication sample & $N=14$ & $\mathrm{n} / \mathrm{a}$ & $N=17$ & \\
\hline Age (mean years, SD) & $27.3(7.5)$ & & $31(10.7)$ & $t(29)=1.17, P=0.25$ \\
\hline Gender (number, \% female) & $10(7 \mid)$ & & $10(58)$ & Chi-Sq $=0.53, t=0.46$ \\
\hline LSAS score (mean, SD) & $86.7(\mid 8.1)$ & & $7.8(5.3)$ & $t(29)=|7|,. P<0.000 \mid$ \\
\hline LSAS score pre (mean, SD) & $85.8(15.3)$ & & $7.7(6)$ & $t(17)=12.7, P<0.000 \mid$ \\
\hline LSAS score post (mean, SD) & $44.5(25.3)$ & & $8.25(8.1)$ & $t(17)=3.6, P=0.0004$ \\
\hline LSAS score post-pre (mean, SD) & $-41.3(28.9)$ & & $0.57(3.4)$ & $t(17)=3.4, P=0.003$ \\
\hline
\end{tabular}

Abbreviations: LSAS, Liebowitz Social Anxiety Scale; n/a, not applicable.

condition (Figure 1a; see Supplementary Figure 2 for results from all conditions). The accuracy decreased to chance performance levels when more than three features were included in the feature set, indicating that additional features were irrelevant to SAD diagnosis. This is consistent with observed performance degradation of Support Vector Machine (SVM) classifiers when irrelevant features are included in the feature set (Judson et al, 2008). Anatomical display of these two features revealed functional connections between left hippocampus and left temporal pole, and between right anterior middle temporal gyrus and left orbitofrontal cortex (Figure 2b; Table 2A). When comparing classification performance of each feature alone, left hippocampus-left temporal pole was more discriminating than right anterior middle temporal gyrus-left orbitofrontal cortex (AUC $=0.77$ vs 0.68 , data not shown).

Specificity of these features to the SAD diagnosis was tested by classifying SAD vs subjects with PD $(n=16)$. Using only the above two features (no feature selection), an AUC of $0.81, P=0.0001$ uncorrected was achieved in discriminating between SAD and PD patients (Table 2B), suggesting relative specificity of these features to SAD. These two features did not discriminate HCs from $\mathrm{PD}$ (AUC $=0.47$, data not shown).

We note that that this primary sample was not balanced for gender (HCs: 11 males, 8 females; SAD: 2 males, 14 females). We therefore tested whether the top two features identified above could predict gender among the combined group (13 males vs 22 females). Classification was not any greater than chance for this classification $(A U C=0.50)$, verifying that classification performance was not an artifact of a sample mismatched for gender. In addition, there was only a slight decrease in performance after applying multiple regression to each feature and removing the effects of age and gender: original AUC/adjusted AUC $=0.88 / 0.80$.
Importantly, replication of these findings (described more below) was tested in a sample that was matched for age and gender.

In addition to the exploratory, data-driven approach above, we examined FC previously identified to be anomalous in SAD, in particular reduced aINS-dACC (Klumpp et al, 2012) and amygdala-dACC and amygdaladlPFC (Prater et al, 2012) in SAD during fear. Although FC differences were mostly consistent with these studies (particularly with the dACC, see Supplementary Results), including the above connections in addition to the two features identified above, individually or collectively, did not improve classification performance, whereas including only these connections resulted in poorer classification performance $(\mathrm{AUC}=0.53)$. In addition, SAD vs HC classification was also conducted using spatial patterns of activity (ie, beta and contrast estimates, which are considered summary measures of activation in response to each condition), and standard GLM/regression analysis and psychophysiological interaction (PPI) analyses seeding with left hippocampus and left temporal pole were conducted (see Supplementary Figures S3-S5, Supplementary Tables S3 and S4, Supplementary Results and Discussion for further details). In summary, during face processing, significantly greater activation of dorsolateral prefrontal cortex was observed in controls, while greater fusiform activity was observed in SAD. However, diagnostic classification performance using these activation patterns was inferior to classification performance using patterns of large-scale FC.

\section{Discriminating SAD vs HCs in an Independent Replication Sample}

To see how well classification using the top two FC features identified above generalized to new data, these features (left 
a

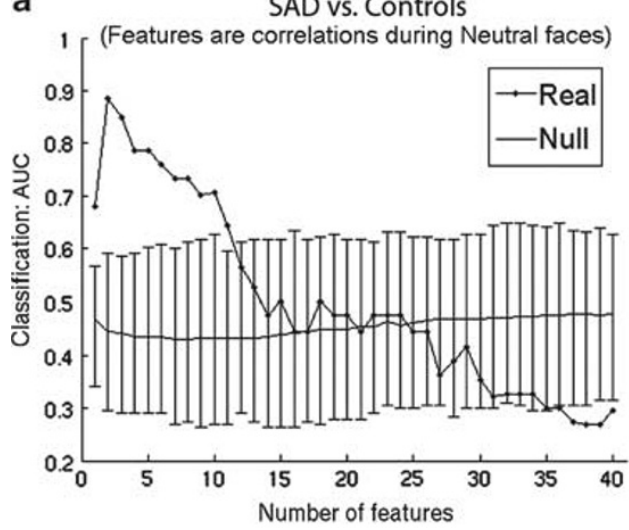

b

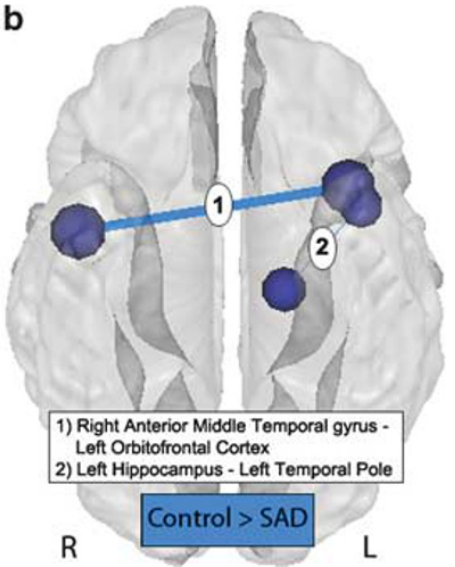

Figure I Functional connectivity that discriminates SAD in primary sample. (a) Classification performance (AUC) when predicting SAD ( $n=16$ ) vs controls $(n=19)$ as a function of the number of features $(1-40)$ included ranked in descending order by their absolute $t$-score. Features were Pearson's correlations using segmented and concatenated time series during the unattended neutral face condition (' $N$ ', black dots, see text for results when using correlations over other stimulus conditions). The peak performance for SAD vs Control classification using ' $\mathrm{N}$ ' correlations (sensitivity $=0.88$, specificity $=0.89, A \cup C=0.89, P<0.002$, corrected) was achieved when learning was based on the top two features in each training set. Mean AUC for shuffled data is plotted along the bottom, with error bars representing $90 \% \mathrm{Cl}$. Ventral (b) anatomical representation of the top two features when classifying SAD vs control subjects using ' $N$ ' correlations. The largest contributing FCs were between R anterior middle temporal gyrus and $L$ orbitofrontal cortex, and $L$ hippocampus and $L$ temporal pole both of which were reduced in SAD (shown in blue). For display purposes, the size of each sphere is scaled according to the sum of the SVM weights of each node's connections. In addition, the thickness of each connection was made proportional to its SVM weight.
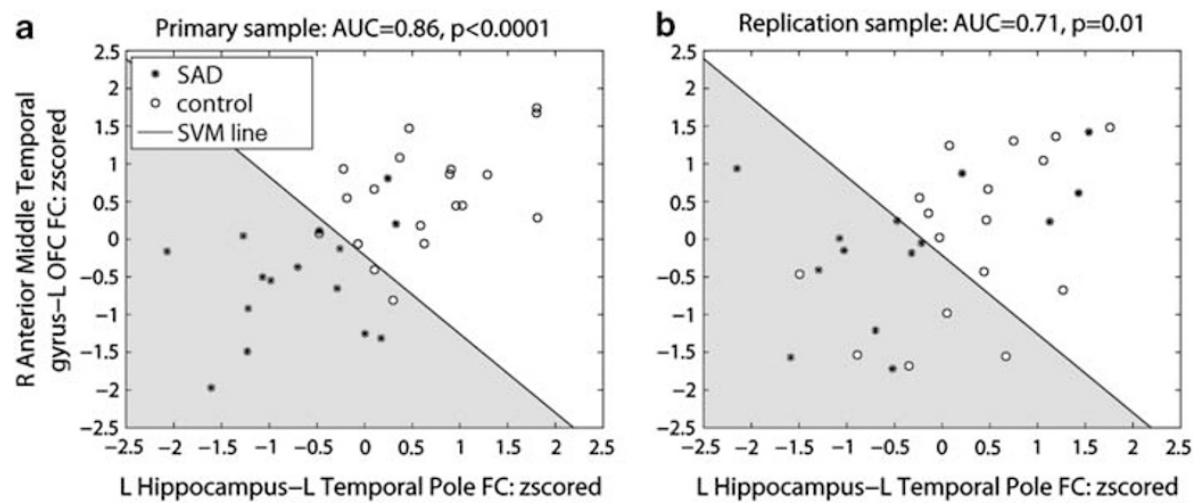

Figure 2 Left-hippocampus-left temporal and left anterior middle temporal gyrus-left orbitofrontal cortex FC predicts SAD in the replication sample. (a) Linear kernel SVM line when learning SAD $(N=16)$ vs Control $(N=19)$ based on the full primary data set using left-hippocampus-left temporal and left anterior middle temporal gyrus-left orbitofrontal cortex FC during unattended neutral faces condition as features. Effects of age and gender were removed, and features were normalized ( $\mathrm{z}$-scored) before learning, so classification performance was slightly lower (AUC $=0.86, P<0.000 \mathrm{I}$ ) than the main text and Figure I. Shaded gray (white) indicates area in which all points were predicted as SAD (control). (b) The same model learned above was used to classify SAD $(N=14)$ vs control $(N=17)$ in the independent replication sample, using left-hippocampus-left temporal and left anterior middle temporal gyrus-left orbitofrontal cortex $F C$ during unattended angry faces ( $A \cup C=0.71, P=0.01$, see main text for results from other conditions).

hippocampus-left temporal pole and right anterior middle temporal gyrus-orbitofrontal cortex) were tested in a second, independent sample of SAD $(n=14)$ vs HCs $(n=17)$. This sample also performed unattended perception of emotional faces, but instead of color identification of fearful and neutral faces (primary sample), they identified the gender of angry, happy, and neutral faces. For this, an SVM model (line) was learned using the full primary data set for the above two features (Figure 2a). To increase generalization ability of the model, features were corrected for the effects of age and gender and z-scored before SVM learning (under these preprocessing steps classification performance was only slightly reduced to $\mathrm{AUC}=0.86$ ). This learned model was applied to the independent replication sample, and the highest AUC was achieved when using FC during angry faces (angry: sensitivity $=0.71$, specificity $=0.71, \mathrm{AUC}=0.71, P=0.01(0.59,0.88) 90 \% \mathrm{CI}$; Figure 2b; Table 3D; happy: $\mathrm{AUC}=0.47, P=0.77$; neutral: $\mathrm{AUC}=0.54, P=0.43$; full: $\mathrm{AUC}=0.57, P=0.31$, data not shown).

Univariate group comparisons over each feature revealed that left hippocampus-left temporal pole FC was significantly reduced in SAD vs HCs, particularly during the angry condition (mean difference $=-0.25, \quad P=0.027$ ) with a trend-significant reduction in the neutral (mean difference $=-0.21, P=0.092$ ) condition (Table 3, first row). Among the cases, this FC did not correlate with symptom severity (as assessed through the Liebowitz Social Anxiety Scale, or LSAS, all $P>0.3$, data not shown). In addition, there were no significant differences observed between SAD and HCs in the right anterior middle temporal gyrus-orbitofrontal cortex FC (all $P>0.2$, data not shown). 
Table 2 (A) Top two FC Features (During Neutral Face Blocks) Discriminating SAD vs HCs, (B) Same Two FC Features (During Neutral Face Blocks) When Predicting SAD vs PD Subjects, and (C) Same Two FC Features (During Angry Faces) predicting SAD vs HCs in an Independent Replication Sample

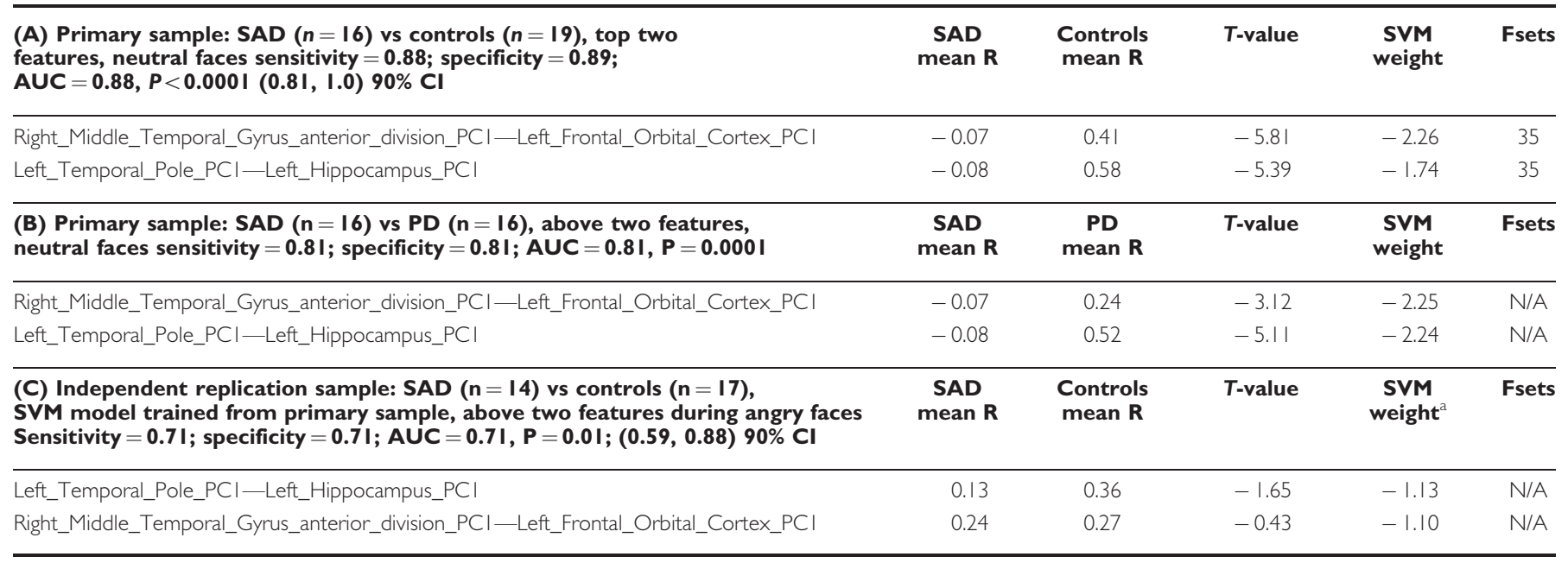

Abbreviations: AUC, Area under the ROC curve; FC, functional connectivity; Fsets, Number of training sets in which the feature was included; PD, Panic Disorder, SAD, Social Anxiety Disorder, SVM, Support Vector Machine.

All reported $P$-values are uncorrected.

aSVM weights were estimated over the full primary sample, and features were adjusted for effects of age and gender and normalized (z-scored) before learning

Table 3 Univariate Statistical Tests of Features Identified in the Primary Sample Tested in a Second, Independent Replication Sample

\begin{tabular}{|c|c|c|c|c|c|c|c|c|}
\hline \multirow[t]{2}{*}{ Left hippocampus-left temporal pole } & \multicolumn{2}{|c|}{ Angry } & \multicolumn{2}{|c|}{ Happy } & \multicolumn{2}{|c|}{ Neutral } & \multicolumn{2}{|c|}{ Full } \\
\hline & Effect size & P-value & Effect size & P-value & Effect size & P-value & Effect size & P-value \\
\hline SAD pre $>$ post $(n=12)$ & -0.414 & 0.007 & -0.343 & 0.039 & -0.245 & 0.098 & -0.343 & 0.036 \\
\hline
\end{tabular}

\section{Changes in Left Hippocampus-Left Temporal Pole FC Following the Treatment}

We examined whether left hippocampus-left temporal pole FC could be considered as a possible biomarker for treatment effects. A subset of the replication sample completed an additional scan following 8 weeks of paroxetine treatment (SAD $n=12$ ). At the group level, social anxiety symptom severity (LSAS) was significantly reduced following the 8-week SSRI (paroxetine) treatment (cases (pre-post) $>$ controls (pre-post) $t_{(17)}=3.4, P=0.003$, Table 1B). Pre minus post comparisons within the SAD subject group $(n=12)$ revealed the left hippocampus-left temporal pole FC, particularly during angry faces, increased following the treatment (angry pre $>$ post, mean change in $R=-0.41, t_{(11)}=-2.9, P=0.007$ paired $t$-test, Table 3, second row). Thus, a paired $t$-test within only the SAD group reveals that this FC increased significantly following the 8-week treatment.

When pre-post $\triangle$ LSAS $v s$ post-pre $\Delta \mathrm{FC}$ (left hippocampus-left temporal pole) was correlated among only SAD cases $(n=12)$, positive, yet non-significant, correlations were observed (angry: $R=0.31, P=0.16$, happy: $R=0.38$, $P=0.11$, neutral: $R=0.16, P=0.31$, full: $R=0.11, P=0.36$, data not shown). Provisionary analyses of pre-post $\Delta$ LSAS $v s$ post-pre $\Delta \mathrm{FC}$ correlations that combined both cases and seven HCs (ie, correlating longitudinal symptom change with change in FC regardless of treatment status) are presented in Supplementary Results. In addition, left hippocampus-left temporal pole FC at baseline was not associated with $\triangle$ LSAS symptom improvement (all $P>0.2$, data not shown). Change in right anterior middle temporal gyrus-left OFC FC did not correlate with change in symptom severity (angry: $R=-0.10, P=0.34$, happy: $R=-0.20, P=0.2$, neutral: $R=0.04, P=0.44$ ). Additional analyses suggest decreases in activation in left hippocampus and left temporal pole in response to angry and neutral faces following the treatment, although effects were subthreshold (see Supplementary Results).

\section{DISCUSSION}

In the current work, a novel and exploratory approach based on multivariate pattern analysis of large-scale, condition-dependent FC (Pantazatos et al, 2012b) was used to identify FC that discriminated individual subjects with SAD. FC features that discriminated SAD from HCs in the primary sample also discriminated SAD from HCs and from subjects with the closely related diagnosis of PD with significant sensitivity and specificity. Additionally, following 8 weeks of treatment of SAD with paroxetine, the most 
discriminative FC feature was increased. Results suggest promise for FC-based biomarkers for psychiatric diagnosis and treatment effects.

Related diagnostic classification studies have applied pattern analysis to condition-dependent activation to a particular probe or stimulus relevant to the disorder (ie, responses to a sad face to predict depression; Fu et al, 2008). While pattern analysis of activation maps takes into account multivariate interactions among regions, activation maps are usually beta maps (summary statistics of activation) or signal that has been averaged across multiple successive scans. Thus, interactions are at a grosser temporal scale. In contrast, the current approach explicitly takes into account scan-to-scan covariation between regions. Here, pattern analysis of FC was more sensitive and specific in discriminating SAD than was multivariate pattern analysis of activation (when using the canonical HRF to model activity), likely due to the fact that it captures information inherent in the interactions among brain regions. Previous large-scale FC approaches capture this information, but only during resting state (ie, resting-state fMRI BOLD to predict schizophrenia ( $\mathrm{Yu}$ et al, 2013) and age (Dosenbach et al, 2010)). The current approach measures conditiondependent FC (ie, large-scale FC during emotional face viewing), combining the sensitivity of multivariate machine-learning analysis with the advantages of both taskbased and resting-state FC approaches.

A meta-analysis of both PET and fMRI studies of SAD before 2007 showed increased activation in amygdala and insula during negative emotional processing (Etkin and Wager, 2007), while many recent fMRI studies of SAD have applied activation analyses focused on the amygdala, and insula (Yoon et al, 2007; Klumpp et al, 2010; Schmidt et al, 2010) to show increased activation in these areas to intense $v s$ low or negative $v s$ neutral emotional stimuli. In addition, univariate analyses showed decreased activation of the amgydala and increased activation in the vmPFC in response to social threat stimuli following 12 weeks of SSRI treatment (Phan et al, 2013). However, although these univariate approaches can identify areas that respond more or less to a particular stimulus, they ignore interactions, or FC, between regions, which are thought to measure information transfer underlying complex cognitive-emotional processing such as during threat or facial effect perception and appraisal (Friston, 2002).

Although previous studies have demonstrated differences in activation and FC (Ding et al, 2011; Liao et al, 2010; Prater et al, 2012; Hahn et al, 2011; Klumpp et al, 2012) between SAD and HCs, the current work is the first to use FC (and activation) to discriminate SAD vs HC diagnostic membership. The current approach of combining machine learning with large-scale, condition-dependent FC is more exploratory and data driven in identifying FC differences than previously used techniques such as PPI Analysis, which only assess FC with a single, a priori specified, 'seed' region at a time. The additional discrimination of SAD from $\mathrm{PD}$ is particularly notable given that these disorders have a significant overlap of both symptoms and neurobiology, such as amygdala hyperactivation and decreased frontal regulation (Damsa et al, 2009; Rauch et al, 2003).

The most discriminative feature was significantly reduced left hippocampus-left temporal pole FC in SAD, which increased concomitant with improvement following 8 weeks of SSRI treatment. Our finding of left hippocampus-left temporal pole FC during face perception in healthy subjects is consistent with the observation of intrinsic FC between anterior hippocampus and anterior temporal pole in humans and non-human primates (Kahn et al, 2008), and with increased FC between hippocampus and left temporal pole during successful retrieval of memory for face-name associations (Tsukiura et al, 2010). Interestingly, we observed that this FC is reduced in subjects with SAD, particularly during neutral (primary sample) and angry face (replication sample) processing (see Supplementary information for further discussion regarding differences between these samples).

Previous findings indicate that the temporal pole has a role in both social and emotional processes including face recognition and theory of mind (Wong and Gallate, 2012), and memory for face-name pairs (Damasio et al, 1996), and it has been proposed that the temporal pole binds complex, highly processed perceptual inputs to visceral emotional responses (Olson et al, 2007). It is also thought to be involved in access to social knowledge during mentalizing, the implicit attribution of intentions and other mental states (Frith and Frith, 2003). The left hippocampus is a key region for memory (ie, autobiographical memory retrieval) (Spreng and Mar, 2010), and FC between this region and the temporal pole may reflect an integration of stored memory with social knowledge during face perception and mentalizing in healthy subjects. This neural process (left hippocampus-left temporal pole FC) is presumably disrupted or under-utilized in SAD, consistent with observations of deficits in memory and recognition of facial identity in social anxiety (Davis et al, 2011; Pérez-López and Woody, 2001), and excessive self-focused attention and fears of negative evaluation in interpersonal situations (Clark and Wells, 1995). The results suggest that this neural disruption is prominent when processing faces that carry signals of negative evaluation, such as angry faces (Stein et al, 2002; Staugaard, 2010), or neutral faces, which persons with SAD are more likely to respond to as threatening (Yoon and Zinbarg, 2008b). The strongest effects for left hippocampusleft temporal pole FC in the replication sample (case $v s$ control differences and longitudinal changes) were observed during the angry condition, with a trend-significant effect for the neutral face condition. For the primary sample there was no angry face condition, so the FC to angry faces is unknown, but it is possible that stronger effects (relative to neutral face condition) might have been observed in the primary sample as well, had the task included an angry face condition. The apparent discrepancy between samples in the significance level of findings for neutral faces may be due to the limitation of small samples yielding marginal power for these analyses, or could be due to the difference in tasks that required attention focused on gender (replication sample task) or on color (primary sample task). Future studies that include both emotional expressions and types of tasks would be required to clarify this issue.

In the absence of a placebo or comparison therapy group, we cannot infer to what extent changes in left hippocampusleft temporal pole FC were specific to SSRI treatment or to clinical improvement. In a recent PET study in which SAD subjects responded to either placebo or SSRI treatment, 
reduction in (amygdala) brain activity was similar in both groups (Faria et al, 2012). Hence, we cannot rule out that left hippocampus-left temporal pole FC would increase in response to any treatment. From the current longitudinal analysis based on only two time points, we also cannot infer whether the changes in left hippocampus-left temporal pole FC preceded, or instead followed, changes in symptom severity. A future study could be designed to employ mediation analysis to more explicitly test whether changes in left hippocampus-left temporal pole FC mediate changes in symptom severity, or vice versa.

Although baseline (pre-treatment), left hippocampus-left temporal pole FC did not predict outcome in response to 8 weeks of SSRI treatment, power to detect predictors of outcome was limited by limited heterogeneity in outcome, as most of the SAD patients improved during treatment. FC (as well as brain activity features identified in related studies, Ding et al, 2011; Doehrmann et al, 2012), may be a useful pre-biomarker to refine the diagnostic classification of psychiatric disorders and advance the development of personalized treatment approaches. For example, it is possible that left hippocampus-left temporal pole FC could be modulated by particular conditions (eg, social threat stimuli under various cognitive reappraisal strategies) within a single scan session, and the extent of this modulation may then be predictive of treatment outcome. Alternatively FC features might be used to identify targets for direct modification by techniques such as transcranial magnetic stimulation. If the FCs identified in this study are further validated by independent replications, then future studies could examine the clinical features of individuals, regardless of diagnosis, that exhibit these features, who might thus also benefit from treatments designed to modulate this circuitry.

Limitations of this study include that the primary data set was not gender matched, and there were differences in ascertainment, diagnostic assessments, and paradigm (ie, color $v s$ gender identification) with the replication sample. However, the fact that replication was significant despite these differences suggest robustness of our approach, and encourage further refinement of the approach and replication in larger samples. The left hippocampus-left temporal pole FC identified here appears to be symptom severity dependent, and as such may not useful as a marker for early detection or a test of risk in those who are pre-symptomatic. However, the current results suggest that future SAD prodrome longitudinal studies should investigate the utility of pattern analysis of large-scale FC during face processing as an approach to identify putative risk biomarkers. Future studies should also include a larger, longitudinal control group to more accurately assess the reliability of conditiondependent FC measures across multiple time points.

Our primary and replication samples contained similar distributions of race/ethnicity in SAD and HC groups: (African American/White/Hispanic/Asian/Unspecified: primary HCs $3 / 9 / 1 / 5 / 1$, primary SAD $2 / 10 / 3 / 0 / 1$, replication HCs $0 / 14 / 1 / 2$, replication SAD $0 / 10 / 3 / 1 / 0$ ), while the viewed stimuli were all Caucasian (Ekman) faces. Previous studies have demonstrated differences in amygdala responses to Black $v s$ White faces during indirect race evaluation (Phelps et al, 2000), while priming race in biracial observers affects visual search for Black and White faces (Chiao et al, 2006).
Unless future studies with larger, multiracial samples confirm that these findings are robust across gender and race/ethnicity, race/ethnicity of the facial stimuli could pose an obstacle in eventually translating the current findings and methodology toward clinical use. In this case, use of schematic face stimuli could be explored to preclude any confounds due to racial bias (Evans et al, 2008).

Further improvements in the spatial and temporal resolution of fMRI data acquisition should improve the sensitivity/specificity of whole-brain functional-connectivity measures. The use of higher field strength (3T) combined with instrumental choices and technical improvements has recently allowed for whole-brain coverage with $2 \mathrm{~mm}$ isotropic resolution with $\mathrm{TR}=0.7 \mathrm{~s}$ for fMRI (Ugurbil et al, 2013). Furthermore, since structural parcellation introduces arbitrary boundaries between regions (Smith et al, 2011), future methodological improvements could include functional parcellation so that the signal from each node region is adequately captured (Ugurbil et al, 2013). Future studies might also utilize a variety of imaging paradigms that activate different regions, such as speech anticipation, eye gaze (Schneier et al, 2009), and other structural imaging modalities (Liao et al, 2011; Talati et al, 2013), as the best discrimination may ultimately result from combining several paradigms and imaging modalities that tap into various neural facets of the disorder.

\section{FUNDING AND DISCLOSURE}

In the past 2 years, FRS has received an honorarium from GlaxoSmithKline for speaking at two conferences. Neither present a conflict of interest with the present study.

\section{ACKNOWLEDGEMENTS}

SPP had full access to all of the data in the study and takes responsibility for the integrity of the data and the accuracy of the data analysis. We wish to thank Stephen Dashnaw and Andrew Kogan for technical assistance with image acquisition; Aviva Olsavsky and Melissa Sy for assistance with subject recruitment and project management; Xian Zhang, Paul Pavlidis, and Tor Wager for helpful discussion and guidance. Nico Dosenbach provided scripts which aided in the 3D network visualizations using the Caret software. This work was supported by an NIMH predoctoral fellowship (NRSA) F31MH088104-02 (SPP/mentor JH), a K01 DA029598 (National Institute of Drug Abuse) and NARSAD Young Investigator Award (AT), a US Army TARDEC W56HZV-04-P-L (JH) and the Sycamore Fund (FRS). Subject scanning and recruitment was also funded by the following grants: PO1MH60970 (PI: MM Weissman) and R21 MH077976 (PI: FRS).

\section{Author contributions}

Conceived, designed and conducted the experiments: SPP AT. Analyzed the data: SPP. Contributed reagents/materials/analysis tools: JH FRS. Drafted the paper: SPP. Supervised the study: JH FRS. All authors read and approved the final paper. Research was conducted in partial fulfillment of the requirements for a $\mathrm{PhD}$ degree in 
Physiology and Cellular Biophysics at Columbia University (SPP/JH mentor).

\section{REFERENCES}

Chiao JY, Heck HE, Nakayama K, Ambady N (2006). Priming race in biracial observers affects visual search for Black and White faces. Psychol Sci 17: 387-392.

Clark DM, McManus F (2002). Information processing in social phobia. Biol Psychiatry 51: 92-100.

Clark DM, Wells A (1995). A cognitive model of social phobia. In: Social phobia: Diagnosis, assessment, and treatment. Guilford Press: New York, NY.

Damasio H, Grabowski TJ, Tranel D, Hichwa RD, Damasio AR (1996). A neural basis for lexical retrieval. Nature 380: 499-505.

Damsa C, Kosel M, Moussally J (2009). Current status of brain imaging in anxiety disorders. Curr Opin Psychiatry 22: 96-110.

Davis JM, McKone E, Dennett H, O'Connor KB, O'Kearney R, Palermo R (2011). Individual differences in the ability to recognise facial identity are associated with social anxiety. PLoS ONE 6: e28800.

Ding J, Chen H, Qiu C, Liao W, Warwick JM, Duan X et al (2011). Disrupted functional connectivity in social anxiety disorder: a resting-state fMRI study. Magn Reson Imaging 29: 701-711.

Doehrmann O, Ghosh SS, Polli FE, Reynolds GO, Horn F, Keshavan A et al (2012). Predicting treatment response in social anxiety disorder from functional magnetic resonance imaging. Arch Gen Psychiatry 1-11.

Dosenbach NU, Nardos B, Cohen AL, Fair DA, Power JD, Church JA et al (2010). Prediction of individual brain maturity using fMRI. Science (New York, NY) 329: 1358-1361.

Ekman P, Friesen WV (1976). Pictures of Facial Affect. Consulting Psychologists Press: Palo Alto, CA.

Etkin A, Klemenhagen KC, Dudman JT, Rogan MT, Hen R, Kandel ER et al (2004). Individual differences in trait anxiety predict the response of the basolateral amygdala to unconsciously processed fearful faces. Neuron 44: 1043-1055.

Etkin A, Wager TD (2007). Functional neuroimaging of anxiety: a meta-analysis of emotional processing in PTSD, social anxiety disorder, and specific phobia. Am J Psychiatry 164: 1476-1488.

Evans KC, Wright CI, Wedig MM, Gold AL, Pollack MH, Rauch SL (2008). A functional MRI study of amygdala responses to angry schematic faces in social anxiety disorder. Depress Anxiety 25: 496-505.

Faria V, Appel L, Åhs F, Linnman C, Pissiota A, Bani M et al (2012). Amygdala subregions tied to SSRI and placebo response in patients with social anxiety disorder. Neuropsychopharmacology 37: 2222-2232.

Friston K (2002). Functional integration and inference in the brain. Prog Neurobiol 68: 113-143.

Frith U, Frith CD (2003). Development and neurophysiology of mentalizing. Philos Trans R Soc Lond B Biol Sci 358: 459-473.

Fu CH, Mourao-Miranda J, Costafreda SG, Khanna A, Marquand AF, Williams SC et al (2008). Pattern classification of sad facial processing: toward the development of neurobiological markers in depression. Biol Psychiatry 63: 656-662.

Gentili C, Gobbini MI, Ricciardi E, Vanello N, Pietrini P, Haxby JV et al (2008). Differential modulation of neural activity throughout the distributed neural system for face perception in patients with social phobia and healthy subjects. Brain Res Bull 77: 286-292.

Gordon E, Koslow S (2010). Integrative Neuroscience and Personalized Medicine. Oxford University Press: New York, NY.

Hahn A, Stein P, Windischberger C, Weissenbacher A, Spindelegger C, Moser E et al (2011). Reduced resting-state functional connectivity between amygdala and orbitofrontal cortex in social anxiety disorder. Neuroimage 56: 881-889.
Jefferys D (1997). Social phobia. The most common anxiety disorder. Aust Fam Physician 26: 1061-1064.

Judson R, Elloumi F, Setzer RW, Li Z, Shah I (2008). A comparison of machine learning algorithms for chemical toxicity classification using a simulated multi-scale data model. BMC Bioinformatics 9: 241 .

Kahn I, Andrews-Hanna JR, Vincent JL, Snyder AZ, Buckner RL (2008). Distinct cortical anatomy linked to subregions of the medial temporal lobe revealed by intrinsic functional connectivity. J Neurophysiol 100: 129-139.

Kessler RC, Berglund P, Demler O, Jin R, Merikangas KR, Walters EE (2005). Lifetime prevalence and age-of-onset distributions of DSM-IV disorders in the National Comorbidity Survey Replication. Arch Gen Psychiatry 62: 593-602.

Kessler RC, McGonagle KA, Zhao S, Nelson CB, Hughes M, Eshleman S et al (1994). Lifetime and 12-month prevalence of DSM-III-R psychiatric disorders in the United States. Results from the National Comorbidity Survey. Arch Gen Psychiatry 51: 8-19.

Klumpp H, Angstadt M, Nathan PJ, Phan KL (2010). Amygdala reactivity to faces at varying intensities of threat in generalized social phobia: an event-related functional MRI study. Psychiatry Res 183: 167-169.

Klumpp H, Angstadt M, Phan KL (2012). Insula reactivity and connectivity to anterior cingulate cortex when processing threat in generalized social anxiety disorder. Biol Psychol 89: 273-276.

Lampe L, Slade T, Issakidis C, Andrews G (2003). Social phobia in the Australian National Survey of Mental Health and Well-Being (NSMHWB). Psychol Med 33: 637-646.

Liao W, Chen H, Feng Y, Mantini D, Gentili C, Pan Z et al (2010). Selective aberrant functional connectivity of resting state networks in social anxiety disorder. Neuroimage 52: 1549-1558.

Liao W, Xu Q, Mantini D, Ding J, Machado-de-Sousa JP, Hallak JE et al (2011). Altered gray matter morphometry and resting-state functional and structural connectivity in social anxiety disorder. Brain Res 1388: 167-177.

Lochner C, Mogotsi M, du Toit PL, Kaminer D, Niehaus DJ, Stein DJ (2003). Quality of life in anxiety disorders: a comparison of obsessive-compulsive disorder, social anxiety disorder, and panic disorder. Psychopathology 36: 255-262.

Mohlman J, Carmin CN, Price RB (2007). Jumping to interpretations: social anxiety disorder and the identification of emotional facial expressions. Behav Res Ther 45: 591-599.

Olson IR, Plotzker A, Ezzyat Y (2007). The Enigmatic temporal pole: a review of findings on social and emotional processing. Brain 130(Pt 7): 1718-1731.

Otto MW, Pollack MH, Maki KM, Gould RA, Worthington 3JJ, Smoller JW et al (2001). Childhood history of anxiety disorders among adults with social phobia: rates, correlates, and comparisons with patients with panic disorder. Depress Anxiety 14: 209-213.

Pantazatos SP, Talati A, Pavlidis P, Hirsch J (2012a). Cortical functional connectivity decodes subconscious, task-irrelevant threat-related emotion processing. Neuroimage 61: 1355-1363.

Pantazatos SP, Talati A, Pavlidis P, Hirsch J (2012b). Decoding unattended fearful faces with whole-brain correlations: an approach to identify condition-dependent large-scale functional connectivity. PLoS Comput Biol 8: e1002441.

Phan KL, Coccaro EF, Angstadt M, Kreger KJ, Mayberg HS, Liberzon I et al (2013). Corticolimbic brain reactivity to social signals of threat before and after sertraline treatment in generalized social phobia. Biol Psychiatry 73: 329-336.

Phelps EA, O'Connor KJ, Cunningham WA, Funayama ES, Gatenby JC, Gore JC et al (2000). Performance on indirect measures of race evaluation predicts amygdala activation. J Cogn Neurosci 12: 729-738.

Prater KE, Hosanagar A, Klumpp H, Angstadt M, Phan KL (2012). Aberrant amygdala-frontal cortex connectivity during 
Diagnostic classification of social anxiety disorder using $\mathbf{f M R I}$

SP Pantazatos et al

perception of fearful faces and at rest in generalized social anxiety disorder. Depress Anxiety 30: 234-241.

Pérez-López JR, Woody SR (2001). Memory for facial expressions in social phobia. Behav Res Ther 39: 967-975.

Randall CL, Thomas S, Thevos AK (2001). Concurrent alcoholism and social anxiety disorder: a first step toward developing effective treatments. Alcohol Clin Exp Res 25: 210-220.

Rapee RM, Heimberg RG (1997). A cognitive-behavioral model of anxiety in social phobia. Behav Res Ther 35: 741-756.

Rauch SL, Shin LM, Wright CI (2003). Neuroimaging studies of amygdala function in anxiety disorders. Ann NY Acad Sci 985: $389-410$.

Schmidt S, Mohr A, Miltner WH, Straube T (2010). Taskdependent neural correlates of the processing of verbal threatrelated stimuli in social phobia. Biol Psychol 84: 304-312.

Schneier FR, Heckelman LR, Garfinkel R, Campeas R, Fallon BA, Gitow A et al (1994). Functional impairment in social phobia. J Clin Psychiatry 55: 322-331.

Schneier FR, Kent JM, Star A, Hirsch J (2009). Neural circuitry of submissive behavior in social anxiety disorder: a preliminary study of response to direct eye gaze. Psychiatry Res 173: 248-250.

Schneier FR, Pomplun M, Sy M, Hirsch J (2011). Neural response to eye contact and paroxetine treatment in generalized social anxiety disorder. Psychiatry Res 194: 271-278.

Schulz C, Mothes-Lasch M, Straube T (2013). Automatic neural processing of disorder-related stimuli in social anxiety disorder: faces and more. Front Psychol 4: 282.

Smith SM, Miller KL, Salimi-Khorshidi G, Webster M, Beckmann CF, Nichols TE et al (2011). Network modelling methods for FMRI. NeuroImage 54: 875-891.

Spreng RN, Mar RA (2010). I remember you: a role for memory in social cognition and the functional neuroanatomy of their interaction. Brain Res 1428: 43-50.

Staugaard SR (2010). Threatening faces and social anxiety: a literature review. Clin Psychol Rev 30: 669-690.

Stein MB, Goldin PR, Sareen J, Zorrilla LT, Brown GG (2002). Increased amygdala activation to angry and contemptuous faces in generalized social phobia. Arch Gen Psychiatry 59: 1027-1034.
Talati A, Pantazatos SP, Schneier FR, Weissman MM, Hirsch J (2013). Gray matter abnormalities in social anxiety disorder: primary, replication, and specificity studies. Biol Psychiatry 73: 75-84.

Talati A, Ponniah K, Strug LJ, Hodge SE, Fyer AJ, Weissman MM (2008). Panic disorder, social anxiety disorder, and a possible medical syndrome previously linked to chromosome 13. Biol Psychiatry 63: 594-601.

Tsukiura T, Mano Y, Sekiguchi A, Yomogida Y, Hoshi K, Kambara $\mathrm{T}$ et al (2010). Dissociable roles of the anterior temporal regions in successful encoding of memory for person identity information. J. Cogn Neurosci 22: 2226-2237.

Ugurbil K, Xu J, Auerbach EJ, Moeller S, Vu AT, Lenglet C et al (2013). Pushing spatial and temporal resolution for functional and diffusion MRI in the Human Connectome Project. Neuroimage 80: 80-104.

Vapnik VN (1999). An overview of statistical learning theory. IEEE Trans Neural Netw 10: 988-999.

Veit R, Flor H, Erb M, Hermann C, Lotze M, Grodd W et al (2002). Brain circuits involved in emotional learning in antisocial behavior and social phobia in humans. Neurosci Lett 328: 233-236.

Wong C, Gallate J (2012). The function of the anterior temporal lobe: a review of the empirical evidence. Brain Res 1449: 94-116. Yoon KL, Fitzgerald DA, Angstadt M, McCarron RA, Phan KL (2007). Amygdala reactivity to emotional faces at high and low intensity in generalized social phobia: a 4-Tesla functional MRI study. Psychiatry Res 154: 93-98.

Yoon KL, Zinbarg RE (2008a). Interpreting neutral faces as threatening is a default mode for socially anxious individuals. J Abnorm Psychol 117: 680-685.

Yoon KL, Zinbarg RE (2008b). Interpreting neutral faces as threatening is a default mode for socially anxious individuals. J Abnorm Psychol 117: 680-685.

Yu Y, Shen H, Zhang H, Zeng LL, Xue Z, Hu D (2013). Functional connectivity-based signatures of schizophrenia revealed by multiclass pattern analysis of resting-state fMRI from schizophrenic patients and their healthy siblings. Biomed Eng Online 12: 10 .

Supplementary Information accompanies the paper on the Neuropsychopharmacology website (http://www.nature.com/npp) 